\title{
Una contribución a la determinación y la influencia de la adherencia entre ladrillos sílico-calcáreos y morteros
}

Tonindustrie-Ztg., $n^{\circ} 2$, febrero 1070 , págs. 50.56

\section{RESUMEN}

Para determinar la adherencia se aplicó un método con el que es posible medir la rotura sin momento flector en muestras hechas de ladrillo aparejados en cruz. Se investigó la influencia de diferentes mezclas de mortero y se encontró que la mejor adherencia se obtenía para una proporción de cal-cemento-arena de 1:2:8. Se determinó también la influencia de la composición granulométrica del árido, del contenido de agua y de la humedad del ladrillo. Las investigaciones se realizaron tanto sobre ladrillos tomados de la producción industrial como sobre ladrillos fabricados en el laboratorio. En el laboratorio se varió la presión de compactado, a igual composición granulométrica de la arena, entre 36 y $725 \mathrm{kp} / \mathrm{cm}^{2}$ y con una presión de compactación de $217 \mathrm{kp} / \mathrm{cm}^{2}$, variando la composición granulométrica sobre un total de 9 muestras. La porosidad total y las porosidades parciales, determinadas mediante porosímetro de presión de mercurio juntas, permiten obtener conclusiones sobre el comportamiento a la adherencia. Una porosidad parcial, medida con el porosímetro de mercurio, indica un porcentaje de la porosidad total, y su índice señala el radio de poros de tamaño de poros inferior en $\AA$. Elevadas resistencias en la adherenciá (alrededor de $1 \mathrm{kp} / \mathrm{cm}^{2}$ ) se consiguen con porosidad total lo más grande posible y una porosidad parcial $\mathrm{P}_{100.000}=30$ a $50 \%$. Para ello tiene que ser reducida la proporción de grano fino $(<0,063 \mathrm{~mm})$ y aproximadamente igual de grande la proporción de grano mediano $(0,063-0,63 \mathrm{~mm})$ y de grano grueso $(>0,63 \mathrm{~mm})$.

La caracterización de la fuerza adhesiva puede hacerse mejor determinando la superficie especifíca según BET. De acuerdo con esto, se consiguen adherencias de alrededor de 1 $\mathrm{kp} / \mathrm{cm}^{2}$ de ladrillos con superficies comprendidas entre 5 y $10 \mathrm{~m}^{2} / \mathrm{g}$. Se llegó también a clasificar 21 clases de ladrillos industriales empleando esta correlación entre el área superficial y la adherencia.

\section{INTRODUCCION}

Experiencias prácticas permiten suponer que, en primer lugar, las propiedades físicas del ladrillo sílico-calcáreo influyen en la adherencia entre ladrillo y mortero y que, en segundo lugar, se encuentran las propiedades del mismo mortero. Sobre esto informó en resumen Ohnemüller (1) y se estableció un catálogo de los factores que mejoran o perturban la adherencia. 
Diversos análisis, singularmente los realizados por Weigler (2) y Högberg (3), hacen suponer que los siguientes factores poseen cierta importancia y, por lo tanto, se tienen en cuenta en los análisis subsiguientes:

a) Propiedades del ladrillo

- porosidad;

- capacidad de absorción de agua;

- poder absorbente;

- permeabilidad a los gases;

- distribución del radio de los poros;

- composición granulométrica;

- humedad del ladrillo.

b) Propiedades del mortero

- contenido de cal;

- contenido de cemento;

- proporción total de conglomerante;

- distribución granulométrica de las arenas de los morteros;

- contenido de agua (medida del asentamiento, trabajabilidad).

\section{INVESTIGACIONES}

\subsection{Investigaciones previas}

Con el fin de poder analizar la influencia de los distintos tamaños en la adherencia, fue necesario mantener constantes las condiciones de los ensayos y efectuar las investigaciones sólo con ladrillos y morteros que acusan una constancia elevada con respecto a sus propiedades. Por lo tanto, para este estudio se emplearon ladrillos de fabricación propia (instalación de ensayos BVKSI Hannóver).

\subsubsection{Datos característicos}

- $90 \%$ mezcla de arena (composición granulométrica, véase tabla 4);

- $10 \%$ hidróxido cálcico blanco fino;

- $4 \%$ humedad (contenido de agua);

- mezcladora de efecto forzado de $50 \mathrm{l}$;

- carga 80-100 kg = masa para 20 ladrillos $\mathrm{NF}$;

- pesada constante por cada ladrillo;

- 4 horas de endurecimiento a 16 atm.

En una primera serie de ensayos se fabricaron ladrillos con presiones de compactación de 36 a $725 \mathrm{~atm}$.

Para hacer las mezclas de arena se empleó arena gruesa de Leine y como arena fina una mezcla de polvos de cuarzo de Dörentruper.

El mortero se hizo con cal blanca fina, PZ 375 (cemento portland) y arena normalizada I 
y II (proporción 1:2) en el mezclador eléctrico manual con una duración de mezclado de 5 minutos. Las proporciones de mezcla fueron, en partes de peso:

\begin{tabular}{|c|c|c|c|}
\hline cal & : & Cemento & Arena \\
\hline 1 & & 2 & 8 \\
\hline 2 & & 1 & 8 \\
\hline 1 & & 0 & 4 \\
\hline 1 & & 0 & 3 \\
\hline 1 & & 2 & 12 \\
\hline 0 & & 1 & 2 \\
\hline 0 & & 1 & 3 \\
\hline 0 & & 1 & 4 \\
\hline
\end{tabular}

\subsubsection{Ensayo de adherencia}

Con el fin de poder determinar la adherencia de dos cuerpos, generalmente se aplica el ensayo de rotura por tracción pura (1), (2), (3). Para ello, o bien se pega al mortero el gancho de empotramiento y se determina la rotura de la adhesión entre el ladrillo y la capa de mortero o se pegan dos ganchos de empotramiento a dos ladrillos unidos con mortero, de manera que pueden producirse dos roturas de adherencia (fig. 1).
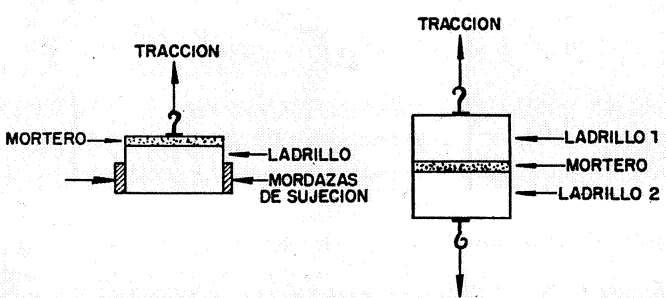

Fig. 1.-Ensayo de rotura ladrillo-mortero.

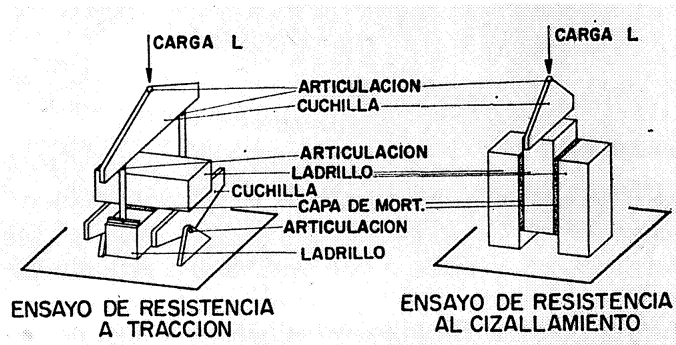

Fig. 2.-Disposición de las probetas en el ensayo de tracción y en el de resistencia al cizallamiento.

Con muchas series de ensayos se mejoró este método basándose en ASTM E 144-59 C, que permite aplicar mortero a igual superficie de ladrillo y garantizar una rotura exenta de momento flector de las probetas. En la figura 2 se ve la disposición esquemática de las muestras y de los ensayos. Se mide la cantidad de mortero necesaria para cada probeta, a fin de obtener un espesor constante de la capa de 9 a $10 \mathrm{~mm}$. Como probetas se aparejan dos ladrillos NF cruzados sobre la superficie de apoyo, de manera que los ejes longitudinales de ambos ladrillos se encuentren en ángulo recto (figs. 3 y 4).

Otra posibilidad de determinación la ofrece el ensayo de cizallamiento. Para ello se aplica mortero a tres ladrillos según la figura 2. La cuchilla colocada en el centro permite realizar el ensayo exento de momento flector.

Las determinaciones hechas en primer lugar en una serie de ladrillos industriales y el 
cálculo de medias aritméticas, desviación standard y coeficientes de variación, permiten las siguientes afirmaciones:

a) la adherencia aumenta en general fuertemente con la edad de las probetas; del $2 .{ }^{\circ}$ al 7. ${ }^{\circ}$ día el aumento es, con arreglo al método de la resistencia a la tracción, de un $50 \%$ y del $7 .^{\circ}$ al $28 .^{\circ}$ día es otra vez de aproximadamente $50 \%$ (fig. 5);

b) ensayos con ladrillos humedecidos con diferente intensidad (secos, con humedad ambiente, mojados, almacenados en agua) no dieron diferencias notables al aplicarles el método de la resistencia a la tracción (fig. 6);

c) en la determinación con arreglo al método de la resistencia al cizallamiento, únicamente acusan un aumento considerable de adherencia con la duración del almacenamiento, las probetas almacenadas en agua y las mojadas; en probetas con otros grados de humedad, después de 7 días se comprueba un máximo de resistencia con descenso posterior (28 días) (fig. 5).

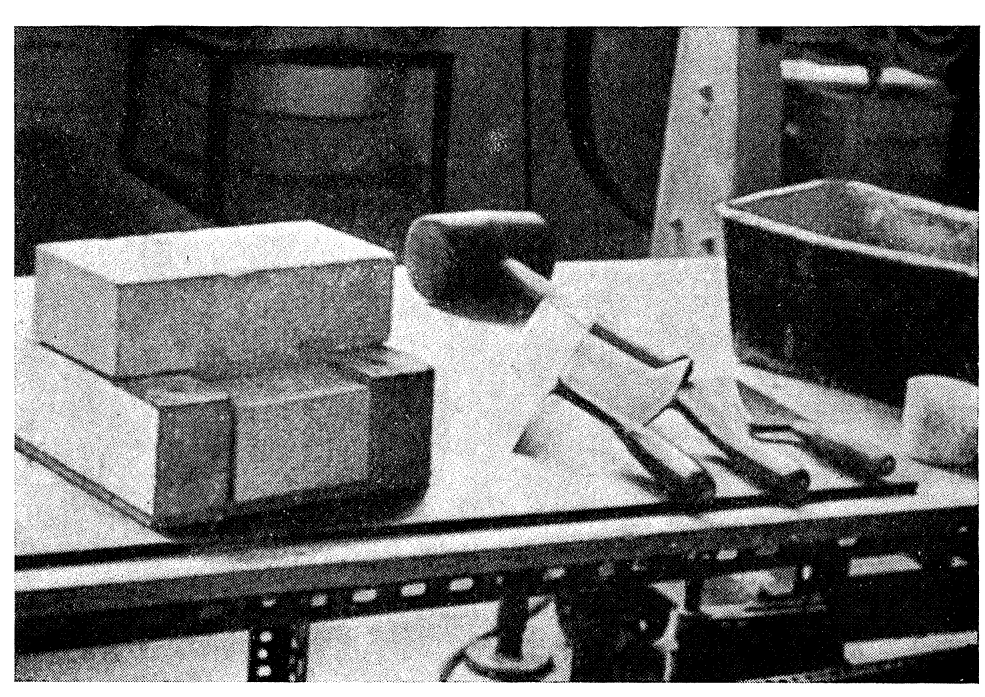

Fig. 3.-Preparación de probetas para el ensayo de resistencia a la tracción.

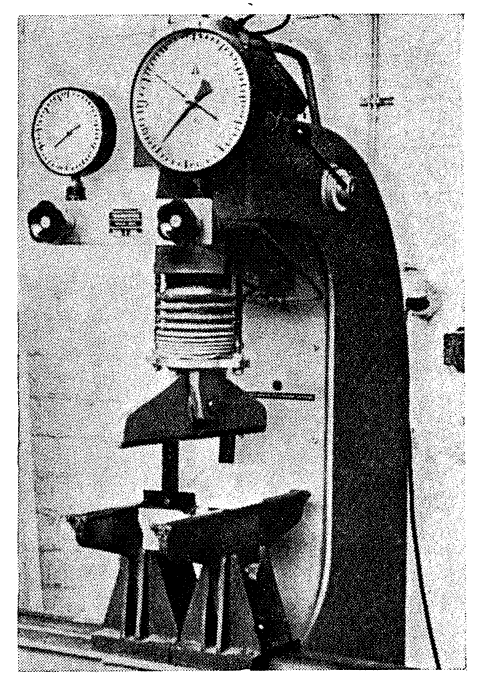

Fig. 4.-Empleo de un aparato de ensayo a flexión para medir la resistencia a tracción.

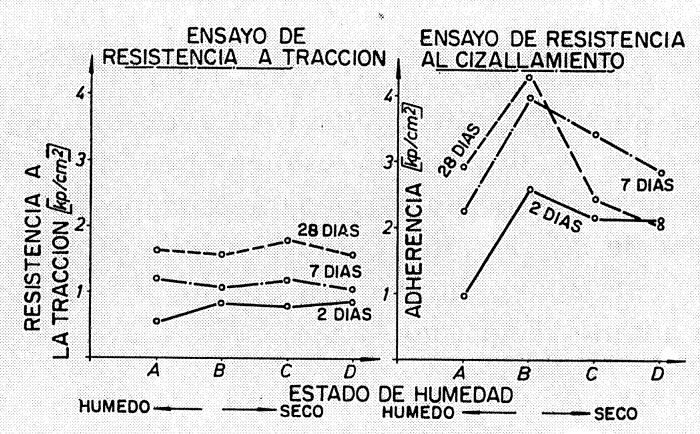

Fig. 5.-Dependencia de la adherencia del estado de humedad después de diversos períodos de conservación.

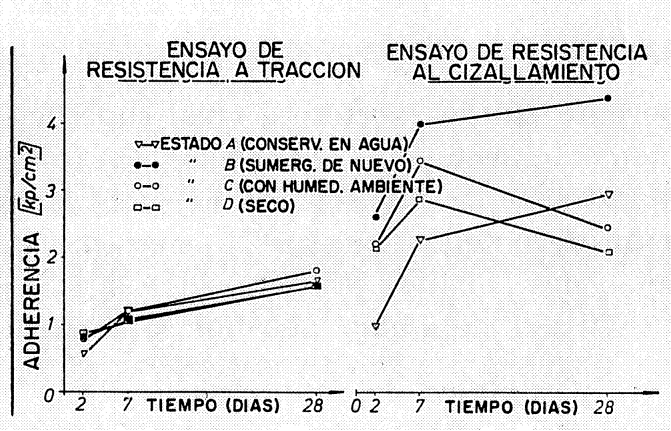

Fig. 6.-Relación entre la adherencia y el tiempo de almacenamiento con diferentes humedades en los ladrillos. 
Al determinarse la adherencia con arreglo al método de resistencia al cizallamiento, existen dos capas de mortero entre tres ladrillos, de manera que son posibles cuatro roturas y siempre se abarca sólo la más débil. En muchos casos se pudieron determinar dos roturas en la adherencia, si después de desprenderse uno de los ladrillos, el cuerpo restante queda todavía entre las mordazas del aparato de ensayo. Los valores son, algunas veces, el doble de los normales.

El método de cizallamiento descrito no se siguió aplicando por lo siguiente:

a) elevado consumo de ladrillos (tres ladrillos en lugar de dos);

b) doble trabajo de mortero;

c) necesidad de tener en cuenta el contenido de humedad (en otros casos no);

d) dos valores de rotura en vez de uno, a veces con gran diferencia;

e) mayor amplitud de oscilación y, en consecuencia, mayor inseguridad en la medición.

Para las series de ensayos principales se determinó:

$\begin{array}{llllllllllllll}- \text { Tipo de ensayo } & \ldots & \ldots & \ldots & \ldots & \ldots & \ldots & \ldots & \ldots & \ldots & \ldots & \ldots & \ldots & \text { resistencia a la tracción. }\end{array}$

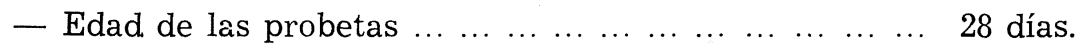

- Estado de los ladrillos al aplicar el mortero ... . . . . . mojado por una inmersión.

O sea, que la adherencia se determina como resistencia a la tracción, y se intenta determinar, de acuerdo con el esquema subsiguiente (fig. 7), las relaciones de los diferentes tamaños entre sí y su influencia en la adherencia.

\subsubsection{Influencia de las propiedades del mortero en la adherencia}

De amplios ensayos preliminares, se deducen las siguientes conclusiones:

1. $\left.{ }^{a}\right)$ El empleo de una arena con una banda granulométrica amplia, de 0 a $2 \mathrm{~mm}$, produce adherencias considerablemente mayores que una arena normal I y II en la relación $1: 2$ mezcladas, ya que esta mezcla contiene prácticamente sólo dos tamaños de grano (tabla 1).

2.a) Cambios en el contenido de agua de amasado del mortero (variación de la medida de asentamiento entre 14 y $22 \mathrm{~cm}$ ) no repercuten tampoco con proporciones de mezcla diferentes (tabla 2, líneas 6 y 7 ).

3. a) Cambios en la proporción de mezcla cal-cemento-arena (tabla 2, línea 5) demuestran que un mortero de cal-arena proporciona adherencias pequeñas, y que morteros de cal-cemento-arena dan adherencias aproximadamente cinco veces mayores. Evidentemente, no desempeña ningún papel importante el empobrecimiento debido a proporción elevada de arena.

Se confirmaron así, en parte, los numerosos ensayos de Högberg (3). En algunos casos, por ejemplo en la determinación de la influencia de diferentes contenidos de agua de amasado del mortero, se obtuvieron resultados discrepantes. Evidentemente, Högberg no ha trabajado con ladrillos definidos, sino con ladrillos industriales, cuyos índices pueden oscilar mucho, precisamente en ladrillos sílico-calcáreos, incluso dentro de series de ladrillos iguales.

Högberg ha conseguido un notable aumento de la adherencia con mayor proporción de arena en el mortero. En los ensayos realizados aquí se prescindió, sin embargo, de sobre- 
pasar la proporción conglomerante-arena de 3:8. En todas las series de ensayos de la investigación principal, se empleó una proporción de cal-cemento-arena de 1:2:8.

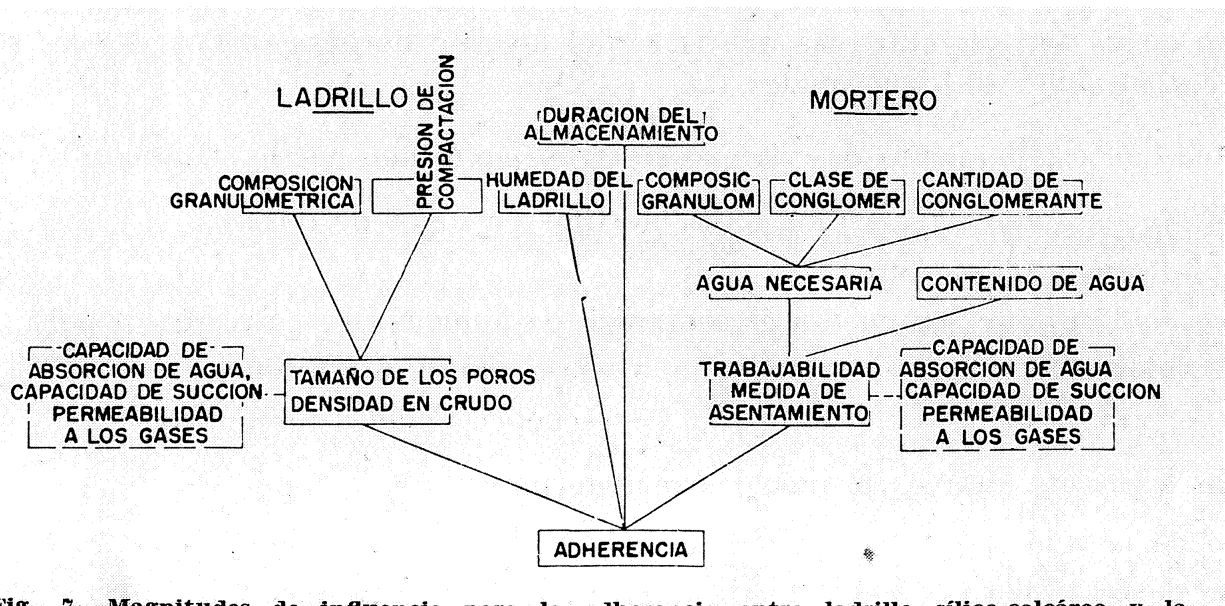

Fig. 7.-Magnitudes de influencia para la adherencia entre ladrillo sílico-calcáreo y la capa de mortero.

Se renunció a preparar una arena de banda granulométrica amplia, en favor de una constancia seguramente mayor de la composición granulométrica utilizando arena normalizada I y II. El consumo de agua de amasado se mantuvo constante y se ajustó a una medida de asentamiento de 18 centímetros:

T A B L A 1

Indices de morteros y ladrillos de dos series de ensayos (ladrillo industrial)

\begin{tabular}{|c|c|c|}
\hline 1. Clases de arena & Banda $\underset{\text { amplia }}{\text { granulométrica }}$ & $\begin{array}{l}\text { Banda granulométrica } \\
\text { estrecha }\end{array}$ \\
\hline 2. Mezcla & \multicolumn{2}{|c|}{ Cal-cemento-arena } \\
\hline 3. Proporción & $1:$ & $2: 8$ \\
\hline 4. Medida de asentamiento & 18 & $\mathrm{~cm}$ \\
\hline 5. Tipo de ladrillo & Ladrillo industiral de & la misma serie \\
\hline 6. Observación & La misma toma de $n$ & uestras en la prensa \\
\hline 7. Superficie de los ladrillos & Ner & ada \\
\hline 8. Humedad & Una inme & sión en agua \\
\hline 9. Número de pruebas & 10 & 8 \\
\hline 10. Adherencia $\left(\mathrm{kp} / \mathrm{cm}^{2}\right)$ & 1,27 & 0,82 \\
\hline 11. Desviación standard & $\pm 0,14$ & $\pm 0,14$ \\
\hline 12. Error relativo $(\%)$ & 11 & 17 \\
\hline
\end{tabular}


T A B L A 2

Indices de morteros y ladrillo de laboratorio

\begin{tabular}{|c|c|c|c|c|c|c|c|c|c|c|}
\hline & Serie de ensayos & 41 & 42 & 43 & 44 & 45 & 46 & 47. & 48 & 49 \\
\hline & Clase de ladrillo & \multicolumn{9}{|c|}{ 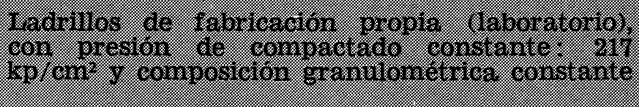 } \\
\hline & Humedad del ladrillo & \multicolumn{9}{|c|}{ Sumergido una vez en agua } \\
\hline 4. & Arena del mortero & Are & $\mathrm{n}$ & rmal & 18 & I en & la rel & itón & $1: 2$ & \\
\hline 5. & Cal-cemento-arena & 0 & 1. & 2 & 1 & 0 & 4 & 1 & 2 & 8 \\
\hline & Medida de asentamiento & 14 & 18 & 22 & 14 & 18 & 22 & 14 & 18 & 22 \\
\hline 7. & Adherencia & - & 1,6 & 1,8 & 0,24 & 0,3 & 0.28 & 1,3 & 1,5 & 1,5 \\
\hline
\end{tabular}

\subsection{Experiencias principales}

2.2.1. Influencia de la presión de compactación sobre la densidad en crudo y distribución de los radios de los poros

La figura 8 muestra el aumento de la densidad en crudo con la presión de prensado, con un incremento mayor hasta unos $300 \mathrm{kp} / \mathrm{cm}^{2}$. Evidentemente, un mayor aumento de la presión sólo produce un pequeño aumento de la compactación. Es de esperar que la distribución del radio de los poros dependa también de la presión y que vaya paralela al cambio de la porosidad. La porosidad total $\mathrm{P}_{\mathrm{g}}$ (poros abiertos y cerrados) se obtiene de la densidad real y de la densidad aparente. Análisis en numerosos ladrillos de cal-arena dieron una densidad real de $2,60 \pm 0,04 \mathrm{~g} / \mathrm{cm}^{3}$.

$$
\mathrm{P}_{\mathrm{g}}=\frac{2,60-\mathrm{R}}{2,60}(\%)
$$

donde $\mathrm{R}$ es la densidad aparente.

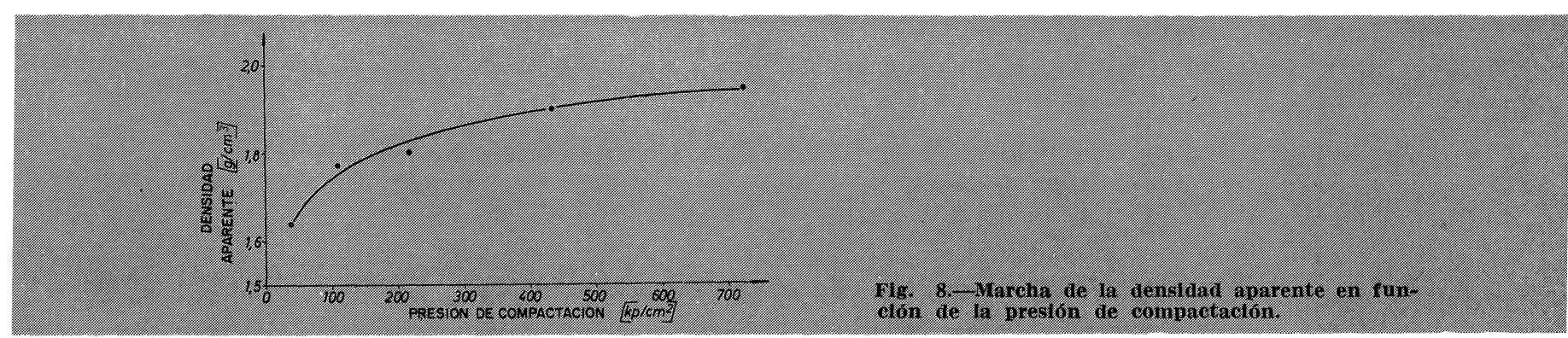

Con esto se obtiene la porosidad total indicada en la tabla 3, columna 4, para los tipos de ladrillo fabricados con diferentes presiones de compactación. 
T A B L A 3

Presión de compactación, densidad aparente y porosidades

\begin{tabular}{|c|c|c|c|c|c|}
\hline $\begin{array}{c}1 \\
\begin{array}{c}\text { Presión de } \\
\text { compactación } \\
\left(\mathbf{k p} / \mathbf{c m}^{2}\right)\end{array}\end{array}$ & $\begin{array}{c}\text { Tipo de } \\
\text { ladrillo }\end{array}$ & $\mathbf{R}\left(\mathrm{g} / \mathrm{cm}^{3}\right)$ & $\mathbf{P}_{\mathrm{g}}(\%)$ & 5 & $\mathbf{P}_{\mathrm{N} 1}$ \\
\hline 36 & 10 & 1,64 & 37 & 34 & 39 \\
\hline 109 & 30 & 1,77 & 32 & 26 & 24 \\
217 & 60 & 1,80 & 31 & 29 & 32 \\
434 & 120 & 1,90 & 27 & 25 & 26 \\
725 & 200 & 1,94 & 25 & 23 & 24 \\
\hline
\end{tabular}

En la columna 5 está indicada la porosidad útil designada con $\mathrm{P}_{\mathrm{N} 1}$, que se consiguió con arreglo al método de inmersión en tetracloruro de carbono. Los valores obtenidos con el porosímetro de presión de mercurio permiten también un cálculo de la porosidad útil. Los valores se indican como $\mathrm{P}_{\mathrm{N} 2}$ en la columna 6. Según este método no se abarcan más que poros con radios comprendidos entre 75 y $550.000 \AA$; pero la confrontación de los valores de la tabla 3 demuestra que todos los poros se hallan dentro de esta gama y que, prácticamente, en esta gama no existen poros cerrados, de manera que es posible una distribución porcentual del espacio poroso total en diferentes clases de tamaños de poros. Una comparación de esta distribución de los radios de los poros en el diagrama es complicada. Por lo tanto, en la figura 9 se representan las proporciones porcentuales de la porosidad en forma de un diagrama bloque, expresándose por poros de los tamaños $<1.000 \AA$, $>1.000 \AA>10.000 \AA \mathrm{y}>100.000 \AA$. Del diagrama bloque se pueden leer inmediatamente los valores para las distintas clases de tamaños de poro:

$\begin{array}{rr}75 \text { hasta } & 1.000 \AA \\ 1.000 \text { hasta } 10.000 \AA \\ 10.000 \text { hasta } 100.000 \AA \\ 100.000 \text { hasta } 550.000 \AA\end{array}$

En la tabla 3 se ve que la porosidad total $\mathrm{P}_{\mathrm{g}}$ disminuye al aumentar la presión de prensado. La porosidad útil $\mathrm{P}_{\mathrm{N} 1}$, o bien $\mathrm{P}_{\mathrm{N} 2}$, para la clase de ladrillo 30 muestra valores demasiado reducidos. Tampoco los valores de la distribución de los radios de los poros ajusta en la tendencia por lo demás inequívoca. Hay que suponer que con esta clase de ladrillo no se cumplen una o varias condiciones de la fabricación (por ejemplo composición granulométrica). Por ejemplo, a pesar de igual porosidad total, una variación en la composición granulométrica puede producir una parte de poros cuyos radios son tan pequeños o tan grandes que no se abarcan con los dos métodos. A un error semejante apunta también la elevada resistencia determinada para la clase de ladrillo 30 . La figura 10 muestra le inequívoca dependencia de la distribución de los radios de los poros de la presión de prensado. Al aumentar la presión de prensado, disminuyen los poros con radios $>1.000 \AA,>10.000 \AA \mathrm{y}>100.000 \AA$. El descenso más fuerte se comprobó para la fracción de poros con radios $>100.000 \AA$. Esta porosidad parcial se designa por $P_{100.000}$ y el valor correspondiente se expreșa en $\%$ de la porosidad total. Así, $\mathrm{P}_{100.000}=45 \%$ indica, 
por ejemplo, que $45 \%$ de la porosidad total se halla constituida por poros cuyo radio es de $100.000 \AA$ y mayor. Parece como si los valores para $\mathrm{P}_{100.000}$ se pueden considerar como magnitud característica y que la adherencia es una función de la porosidad parcial $\mathrm{P}_{100.000}$. En una representación semejante (fig. 10) no se tuvieron en cuenta los valores de la clase 30 , ya que, debido a un error sin aclarar, no se los pudo clasificar. Como quiera que únicamente existen cuatro puntos de medición, esta representación hay que considerarla, en principio, como un esquema. Las dependencias indicadas hay que demostrarlas mediante más series de ensayos.

En el punto A hay un ladrillo que, como consecuencia de una reducida presión de prensado $\left(36 \mathrm{kp} / \mathrm{cm}^{2}\right)$, tiene una proporción elevada $(100.000 \AA)$ de poros y una porosidad elevada (37\%), acusando una reducida adherencia. Con un aumento de la presión de compactación van paralelos el descenso de la porosidad y la proporción de poros grandes, aumentando la adherencia hasta que ha alcanzado un máximo en $\mathrm{B}$. Continuando el aumento de la presión de prensado, bien por no llegar a una porosidad determinada o bien por una proporción determinada de poros grandes, disminuye la adherencia y en el punto $\mathrm{C}$ ( $25 \%$ de porosidad) alcanza el valor más bajo.

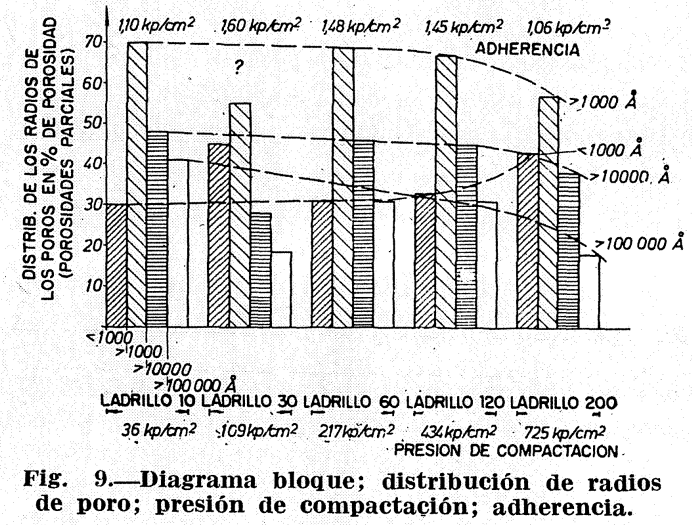

Fig. 9.-Diagrama bloque; distribución de radios
de poro; presión de compactación; adherencia.

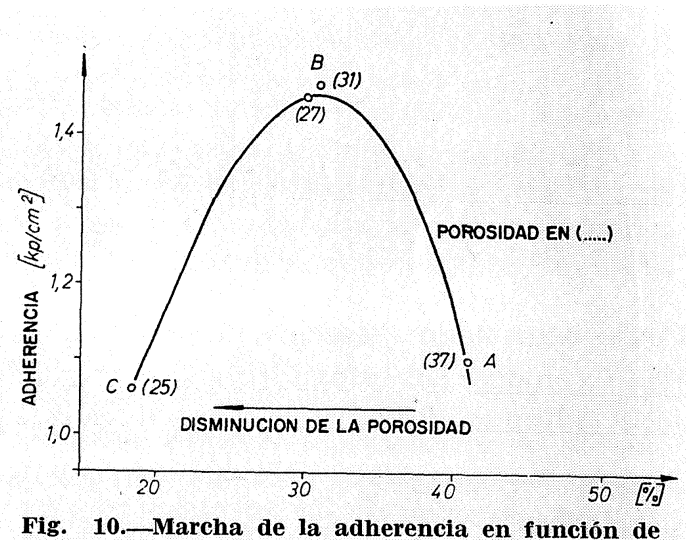

Fig. 10.-Marcha de la adherencia en función de la porosidad parcial $\mathbf{P}_{100,000}$.

Por consiguiente, una buena adherencia presupone un determinado tamaño de poros o bien una determinada distribución del tamaño de los mismos. En la serie de ensayos descrita, las mejores adherencias se consiguieron con $30-35 \%$ para $\mathrm{P}_{100.000}$. Como quiera que $1 \AA=10^{-4} \mu$, se trata aquí de poros con un diámetro $>20 \mu$. Tal diámetro de poros es ya muy grande para ladrillos sílico-calcáreos y debiera de ser el límite superior máximo (1). Pero se obtiene la misma tendencia descrita en la figura 10, si en vez de $P_{100.000}$ se quitan los valores para $P_{10.000} \circ P_{1.000}$. Así se llegaría a diámetros de poros $>2 \mu o$ bien $>0,2 \mu$. Con un diámetro $<0,2 \mu$, desde luego hay que agregar todavía 20 a $30 \%$ de la porosidad total $\mathrm{P}_{\mathrm{g}}$.

Es evidente que la adherencia viene determinada, esencialmente, por la capacidad de succión de los capilares y que esta capacidad la determina el diámetro de los capilares (diámetro capilar). 
T A B L A 4

Composición granulométrica de la arena de diez mezclas de arena

\begin{tabular}{|c|c|c|c|c|c|c|c|c|c|c|}
\hline \multicolumn{11}{|c|}{ Residuos (\%) } \\
\hline Mezcla n.o & 1 & 2 & 3 & 4 & 5 & 6 & 7 & 8 & 9 & 10 \\
\hline$(\mathrm{mm})$ & & & & & & & & & & \\
\hline$>2$ & 9,3 & 4,6 & - & 13,9 & 9,3 & - & 4,6 & 4,6 & - & 1,28 \\
\hline $\begin{array}{ll}2 & -1\end{array}$ & 17,6 & 11,1 & 4,6 & 24,1 & 16,1 & 3,1 & 9,6 & 8,0 & 1,5 & 1,28 \\
\hline $1-0,63$ & 25,2 & 17,5 & 9,8 & 32,9 & 21,9 & 6,5 & 14,2 & 10,8 & 3,3 & 3,95 \\
\hline $0,63-0,4$ & 19,3 & 19,8 & 21,0 & 18,6 & 12,4 & 13,7 & 13,1 & 6,3 & 6,9 & 10,90 \\
\hline $0.4-0,2$ & 23,1 & 37,8 & 51,9 & 8,5 & 5,7 & 34,9 & 20,4 & 2,8 & 17,4 & 56,10 \\
\hline $0,2-0,1$ & 4,8 & 7,7 & 10,5 & 2,0 & 1,8 & 7,5 & 4,7 & 1,7 & 4,5 & 22,30 \\
\hline $0,1-0,063$ & 0,4 & 0,8 & 1,2 & 一 & 2,0 & 2,8 & 2,4 & 4,0 & 4,4 & 2,00 \\
\hline $0,063-0,032$ & 0,3 & 0,7 & 1,0 & - & 8,3 & 8,8 & 8,5 & 16,4 & 16,7 & 2,70 \\
\hline $0,032-0,01$ & - & - & - & - & 13,7 & 13,7 & 13,5 & 27,4 & 27,3 & 0,70 \\
\hline$<0,01$ & - & - & - & - & 9,0 & 9,0 & 9,0 & 18,0 & 18,0 & - \\
\hline Suma & 100,0 & 100,0 & 100,0 & 100,0 & 100,2 & 100,0 & 100,0 & 100,0 & 100,0 & 101,21 \\
\hline
\end{tabular}

Poros demasiado estrechos impiden la succión, lo mismo que ocurre con los poros demasiado grandes. En estos últimos se produciría además una evaporación excesivamente rápida del agua del mortero y conduciría a adherencias demasiado reducidas.

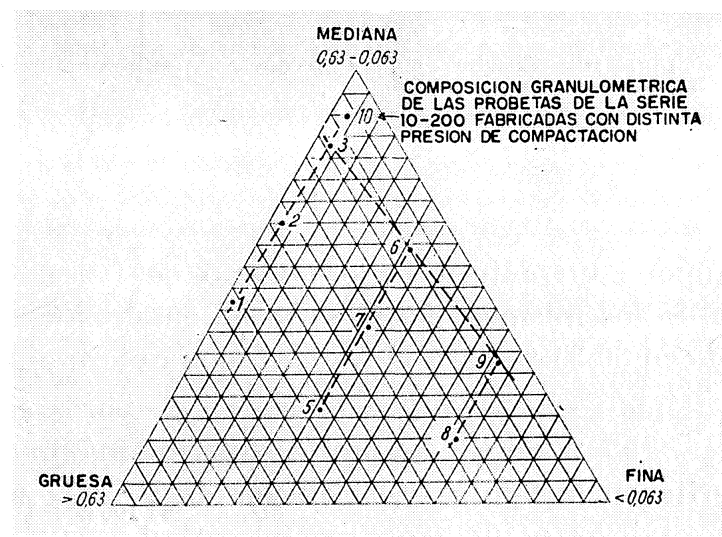

Fig. 11.-Composición granulométrica de diez mezclas de arena.

2.2.2. Influencia de la composición granulométrica en las propiedades de los ladrillos con presión de compactación constante

Es posible que las variaciones en la composición granulométrica influyen de tal modo en la distribución de los poros, que siga aumentando la adherencia y que en una serie de "ensayos semejante se reconocieran funciones (dependencias) todavía mejores. Con este 
fin, se fabricaron nuevas clases de ladrillos con una presión constante de $217 \mathrm{kp} / \mathrm{cm}^{2}$. La distinta composición granulométrica se consiguió con distintas proporciones de mezcla de tres fracciones de arena. La composición granulométrica de las mezclas se indica en la tabla 4. Para una visibilidad mejor, se resumieron los contenidos de varias fracciones y se los designó con gruesa, mediana y fina (tabla 5). Las mezclas se incluyeron en un diagrama triangular (fig. 11), el cual permite considerar los cuatro grupos de mezclas:

$$
\begin{array}{r}
1 / 2 / 3 \\
5 / 7 / 6 \\
8 / 9 \\
3 / 6 / 9
\end{array}
$$

Las probetas de la mezcla 4 se deshicieron, por lo que no fue posible realizar mediciones correctas.

\begin{tabular}{|c|c|c|c|c|c|c|c|c|c|c|c|c|}
\hline \multicolumn{13}{|c|}{ Residuo (\%) } \\
\hline Mezcla n.o & 1 & 2 & 3 & 5 & 7 & 6 & 8 & 9 & 10 & 3 & 6 & 9 \\
\hline \multicolumn{13}{|l|}{$\begin{array}{l}\text { (mm) } \\
\text { "grueso" }\end{array}$} \\
\hline$>0,63$ & 52 & 33 & 14 & 47 & 28 & 10 & 23 & 5 & 7 & 14 & 10 & 5 \\
\hline \multicolumn{13}{|l|}{ "mediano" } \\
\hline $0,63-0,063$ & 48 & 66 & 85 & 22 & 41 & 59 & 15 & 33 & 90 & 85 & 59 & 33 \\
\hline \multicolumn{13}{|l|}{ "fino" } \\
\hline$<0,063$ & 0 & 1 & 1 & 31 & 31 & 31 & 62 & 62 & 3 & 1 & 31 & 62 \\
\hline
\end{tabular}

T А в L A 5

Proporción de los tipos de grano "grueso", "mediano" y "fino", de mezcla cal-arena

Las porosidades reunidas en la tabla 6, así como las adherencias para las mezclas permiten deducir las siguientes conclusiones:

1) Con igual presión de prensado se pueden obtener las mismas porosidades con tamaños de grano extremadamente diferenciados de las mezclas. Compárese las mezclas $1 / 2$ y $8 / 9$.

2) Una confrontación de las adherencias y porosidades muestra que entre ambas magnitudes no existe ninguna dependencia. Para conseguir adherencias elevadas no tiene que existir sólo una gran porosidad, sino que además esta porosidad ha de estar caracterizada por poros con radio determinado. Esto puede realizarse con la poro- 
sidad parcial $\mathrm{P}_{100.000}$ ó $\mathrm{P}_{10.000}$. Esta porosidad parcial será lo más grande posible, es decir, que la proporción de poros formada por poros con radio $\geqslant 10 \mu$ o bien $R \geqslant 1 \mu$ ha de estar entre el 30 y el $50 \%$ de la porosidad total. Por ejemplo, las probetas 5 a 9 tienen porosidades parciales $\mathrm{P}_{100.000}$ de menos de $5 \%$, o sea, que las probetas son de poro muy fino y dan adherencias menores de $0,5 \mathrm{kp} / \mathrm{cm}^{2}$ (tabla 6).

3) Con el fin de conseguir elevada porosidad y buena adherencia ha de ser, en primer lugar, muy reducida la proporción de grano fino de las mezclas y, en segundo lugar, un tamaño aproximadamente igual a la proporción de grano grueso y de mediano (véase tablas 5 y 6 , mezcla 1 ). Un aumento de la proporción de grano mediano a cargo de la proporción de grano grueso conduce a una disminución de la adherencia (mezclas 2 y 3), aunque con valores de alrededor de $1 \mathrm{kp} / \mathrm{cm}^{2}$ todavía es aceptable. Si se observan los valores de la mezcla 10 , hay que suponer que se puede tolerar un aumento en la proporción de grano mediano, si al mismo tiempo se agrega también algo de grano fino. No admiten discusión las mezclas con contenidos de grano fino de alrededor de $30 \%$ y más.

T А в L А 6

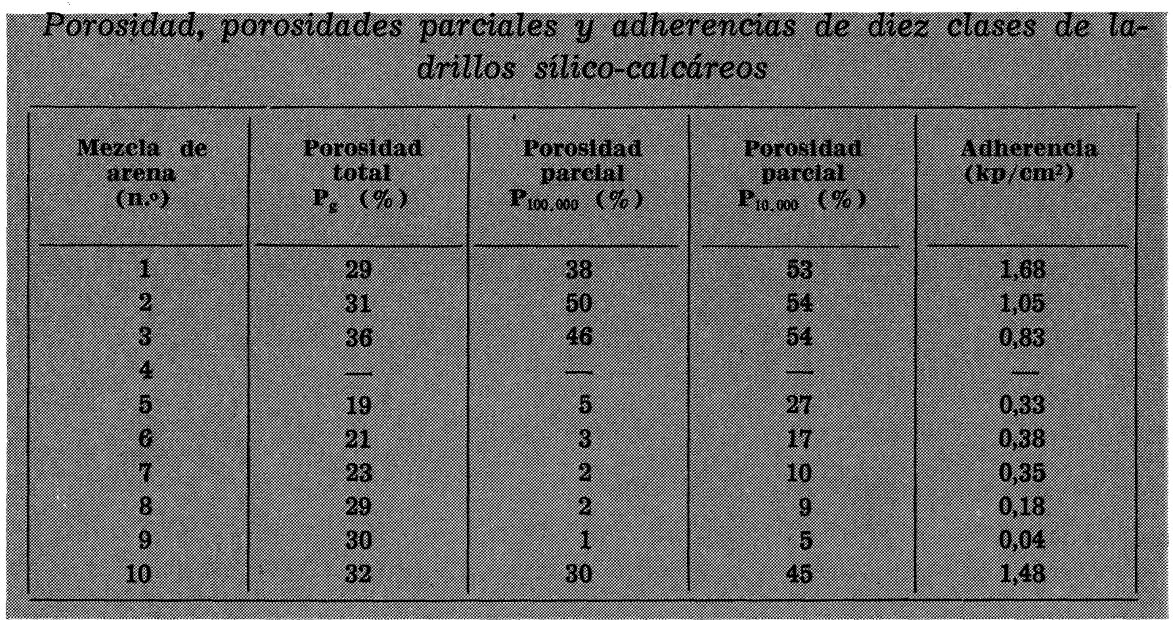

2.2.3. Influencia de la superficie específica de probetas de ladrillo sílico-calcáreo en la adherencia

Amplias investigaciones realizadas, tanto en la Asociación federal de la industria de ladrillos sílico-calcáreos (Hannóver) como en el Instituto para ladrillos y tierras, con respecto a la influencia de la permeabilidad a los gases en diferentes propiedades de ladrillos sílico-calcáreos no han confirmado, desgraciadamente, las dependencias (funciones) esperadas. Tanto el estudio de ladrillos enteros como de probetas sacadas de ellos demostraron una magnífica reproductibilidad para la probeta individual, pero oscilaciones de 100 $\%$ y más dentro de la serie de ladrillos. Claro es que estas oscilaciones fueron mayores en ladrillos industriales que en probetas confeccionadas en el laboratorio; pero también una fabricación esmerada dio todavía grandes oscilaciones, cuyo origen no ha podido aclararse hasta la fecha.

En cambio, la determinación de la superficie específica según BET $\left(\mathrm{m}^{2} / \mathrm{g}\right)$ suministra una afirmación inequívoca con respecto al comportamiento de la adherencia. Esta función se ha representado en la figura 12 para las nueve clases de ladrillos con distinta composición granulométrica del componente arena. 
También una gran parte de 21 clases de ladrillos industriales sigue esta función (dependencia). La figura 13 muestra que los ladrillos industriales se hallan en una zona (gama) de adherencias de 0,5 a $1,0 \mathrm{kp} / \mathrm{cm}^{2}$. Clases de ladrillos con valores fuera del campo rayado poseen una o varias magnitudes de influencia, que discrepan extremadamente de los valores corrientes; por ejemplo, presión de prensado demasiado baja, ninguna proporción de grano fino, contenido muy elevado de $\mathrm{CaO}$. Como quiera que estos valores extremos influyen con muy distinta intensidad en la superficie específica y en la adherencia, hay que suponer también que la superficie no sirve todavía como magnitud única para seguir el comportamiento de la adherencia siendo necesarios más ensayos.

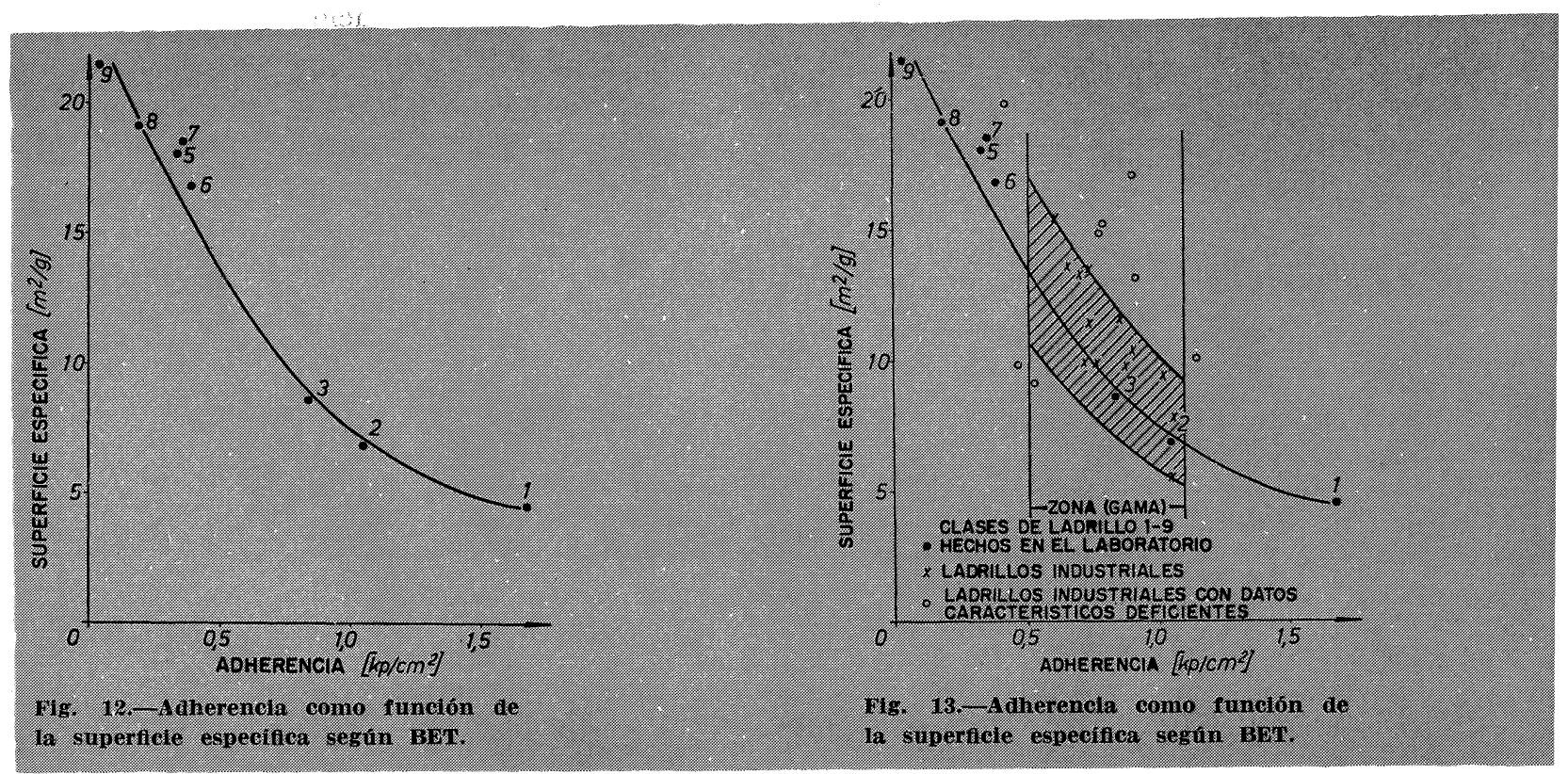

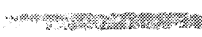

\section{B I B L I O G R A F I A}

(1) OHNemüLleR, W.: Reacciones entre superficie de los ladrillos y mortero. Forschungsberichte des Landes Nordrhein-Westfalen n. ${ }^{\circ} 1.759$.

(2) Weigler, H.: Adherencia del enlucido. Berichte aus der Baufaschung, n. ${ }^{\circ}$ 43, Berlin, 1965.

(3) HögbeRG, E.: Agurre del mortero. Report 40, 1967 fran Byggforskningen, Estocolmo. Publicado por el National Swedish Institute for Building Research. 